\title{
QSAR investigation on benzimidazole derivatives in Trichomonosis' disease
}

\author{
Alireza Nemati Rashtehroodi ${ }^{1}$, Ghasem Ghasemi², \\ ${ }^{1}$ Young Researchers club and elite, Islamic Azad University, Rasht Branch, Rasht, Iran \\ Mr.Alireza.Nemati@gmail.com \\ 2 Department of Chemistry, Islamic Azad University, Rasht Branch, Rasht, Iran \\ Ghasemi@iaurasht.ac.ir
}

\begin{abstract}
Globally trichomoniasis affects approximately 152 million people as of 2010 (2.2\% of the population). It is more common in women $(2.7 \%)$ than males (1.4\%). The American Social Health Association estimates trichomoniasis affects 7.4 million previously unaffected Americans each year and is the most frequently presenting new infection of the common sexually transmitted diseases. On the pattern, QSAR study has been done on benzimidazole derivatives as potent inhibitors with trichomonicidal activity. Genetic algorithm (GA), artificial neural network (ANN), stepwise multiple linear regression (stepwise-MLR) were used to create then on non-linear and linear QSAR models. Geometry optimization of compounds was carried out by B3LYP method employing 6-31G (2d) basis set. HyperChem, Gaussian 03W, and Dragon (version 5.5) software programs were used for geometry optimization of the molecules and calculation of the quantum chemical descriptors. Finally, Unscrambler program was used for the analysis of data. The root-mean square errors of the training set and the test set for GA-ANN model using jack-knife method, were $0.1840,0.5051$ and R2 was 0.70 . Also, the R and $\mathrm{R} 2$ values in the gas phase were obtained $0.78,0.61$ from GA-stepwise MLR model. According to the obtained results, we find out GA-ANN model is the most favorable method toward the other statistical methods. Also, we would suggest that compounds No. 20,33, 58, 48 and 47 as the most appropriate structure for the design of drugs to pharmacists.
\end{abstract}

\section{Indexing terms/Keywords}

Benzimidazole derivatives; QSAR model; Genetic Algorithm; Artificial Neural Network.

\section{Academic Discipline And Sub-Disciplines}

Biotechnology; Organic Chemistry; Inorganic Chemistry and etc.

\section{SUBJECT CLASSIFICATION}

Medicinal Chemistry Subject Classification; 21st Iranian Seminar of Organic Chemistry- Ilam University

\section{TYPE (METHOD/APPROACH)}

Genetic algorithm (GA), artificial neural network (ANN), stepwise multiple linear regression (stepwise-MLR) were used to create then on non-linear and linear QSAR models. QSAR study has been done on benzimidazole derivatives as potent inhibitors with trichomonicidal activity.

\section{Council for Innovative Research}

Peer Review Research Publishing System

\section{Journal: JOURNAL OF ADVANCES IN BIOTECHNOLOGY}

Vol 4 , No. 3

www.ciribt.org , jbteditor@gmail.com 


\section{INTRODUCTION}

Trichomoniasis (or "trich") is a very common sexually transmitted disease (STD) that is caused by infection with a protozoan parasite called Trichomonas vaginalis. Although symptoms of the disease vary, most women and men who have the parasite cannot tell they are infected. Trichomoniasis is considered the most common curable STD. In the United States, an estimated 3.7 million people have the infection, but only about $30 \%$ develop any symptoms of trichomoniasis. Infection is more common in women than in men, and older women are more likely than younger women to have been infected [1]. Trichomoniasis can increase the risk of getting or spreading other sexually transmitted infections. For example, trichomoniasis can cause genital inflammation that makes it easier to get infected with the HIV virus, or to pass the HIV virus on to a sex partner [2]. Benzimidazole is a heterocyclic aromatic organic compound. It is an important pharmacophore and a privileged structure in medicinal chemistry. This compound is bicyclic in nature which consists of the fusion of benzene and imidazole. Nowadays is a moiety of choice which possesses many pharmacological properties [3].Structure-activity modeling plays an important role in government programs in support of protecting human populations from exposure to environmental contaminants [4]. Specifically, computational methods to identify chemicals that may pose endocrine disruption hazard for additional in vitro or in vivo testing are important prioritization approaches [5].QSAR is an effective way of optimizing or correlating the biological activity within congeneric series with certain structural features or with atomic, group or molecular descriptors, such as lipophilicity, polarizability, and electronic and steric properties. Previously, we reported several examples of adopting a QSAR approach to probe the nature of interactions of various classes of ligands towards the cyclooxygenase enzyme [6-12]. QSAR attempts to find consistent relationship between biological activity and molecular properties, so that these "rules" can be used to evaluate the activity of new compounds. Today, QSARs are being applied in many disciplines with much emphasis in drug design. [13], [14].

\section{EXPERIMENTAL}

The structures of the benzimidazole derivatives used in this study [15] (Table 1). The 3D structures of the molecules were generated using the built optimum option of Hyperchem software (version 8.0), Then, the structures were fully optimized based on the ab initio method, using DFT level of theory. Hyperchem, ChemOffice and Dragon (version 5.5) programs were employed to calculate the molecular descriptors. All calculations were performed using Gaussian 03W program series. Geometry optimization of compounds was carried out by B3LYP method employing 6-31G (2d) basis set. In this study, the independent variables were molecular descriptors and the dependent variables were the actual half maximal inhibitory concentration (IC50) values. Overall, more than 3224 theoretical descriptors were selected and calculated. Finally, Unscrambler (version 9.7) program was used for analysis of data and statistical methods. For each compound in the training sets, the correlation equation was derived with the same descriptors. Then, the obtained equation was used to predict log $(1 / \mathrm{IC50})$ values for the compounds from the corresponding test sets. In the present work, the method of stepwise multiple linear regression (stepwise, MLR) to select the most appropriate descriptor of all descriptor was used. Totally 3224 descriptors were generated that were too many to be fitted in our models. So, it was necessary to reduce the number of descriptors through an objective feature selection which was performed in three steps. First, descriptors that had the same value for at least $70 \%$ of compounds within the dataset were removed. In next step, descriptors with correlation coefficients less than 0.4 with the dependent variable were regarded redundant and removed. Finally, since highly correlated descriptors provide approximately identical information, a pair wise correlation was performed. When their correlation coefficient exceeded 0.90 , one of two descriptors was randomly removed.

\section{RESULTS AND DISCUSSIONS}

Based on the types of variable selection method and also the types of the feature mapping technique, these models can be shown as GA-ANN Jack-Knife and cross validation. It revealed that the GA-ANN model was much better than other models is given in Table 2. Considering the experimental error, the overall prediction of the log (1/IC50) values was quite satisfactory.

The efficiency of the QSAR model to predict log (IC50) value was also estimated using the internal cross-validation method, this resulted predictions of the log (1/IC50) using Jack-Knife model is given in Table 3.

In the present study, linear variable selection methods were used to select the most significant descriptors (stepwise MLR) (Table 4).

The six most significant descriptors which were selected by stepwise MLR are as follows:

BLI, RDF090m, G(N..N), MATS5v, RDF035u, HATS1e.

Results from the stepwise MLR method is shown in Figure 2. According to this figure, R2 between the expected values and the observed values were near to a stright line. Also, the $R$ and $R 2$ values were obtained as $0.78,0.61$ from stepwise MLR model. Electronegativities, atomic masses and atomic van der Waals volumes were important descriptors in this study. 
Table1. Structures of benzimidazole derivatives used for QSAR model building

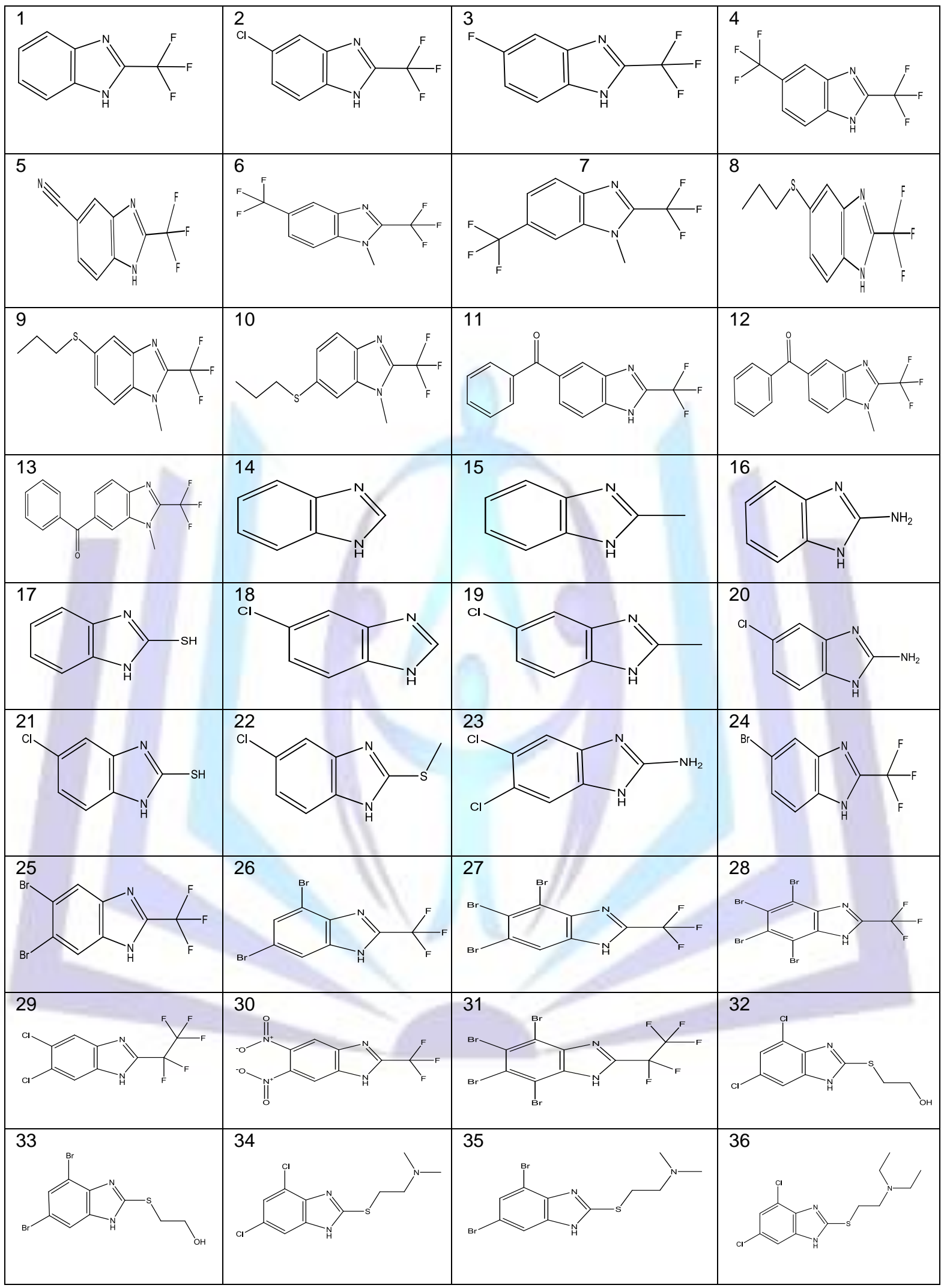


ISSN 2348-6201

Table 1: Continued

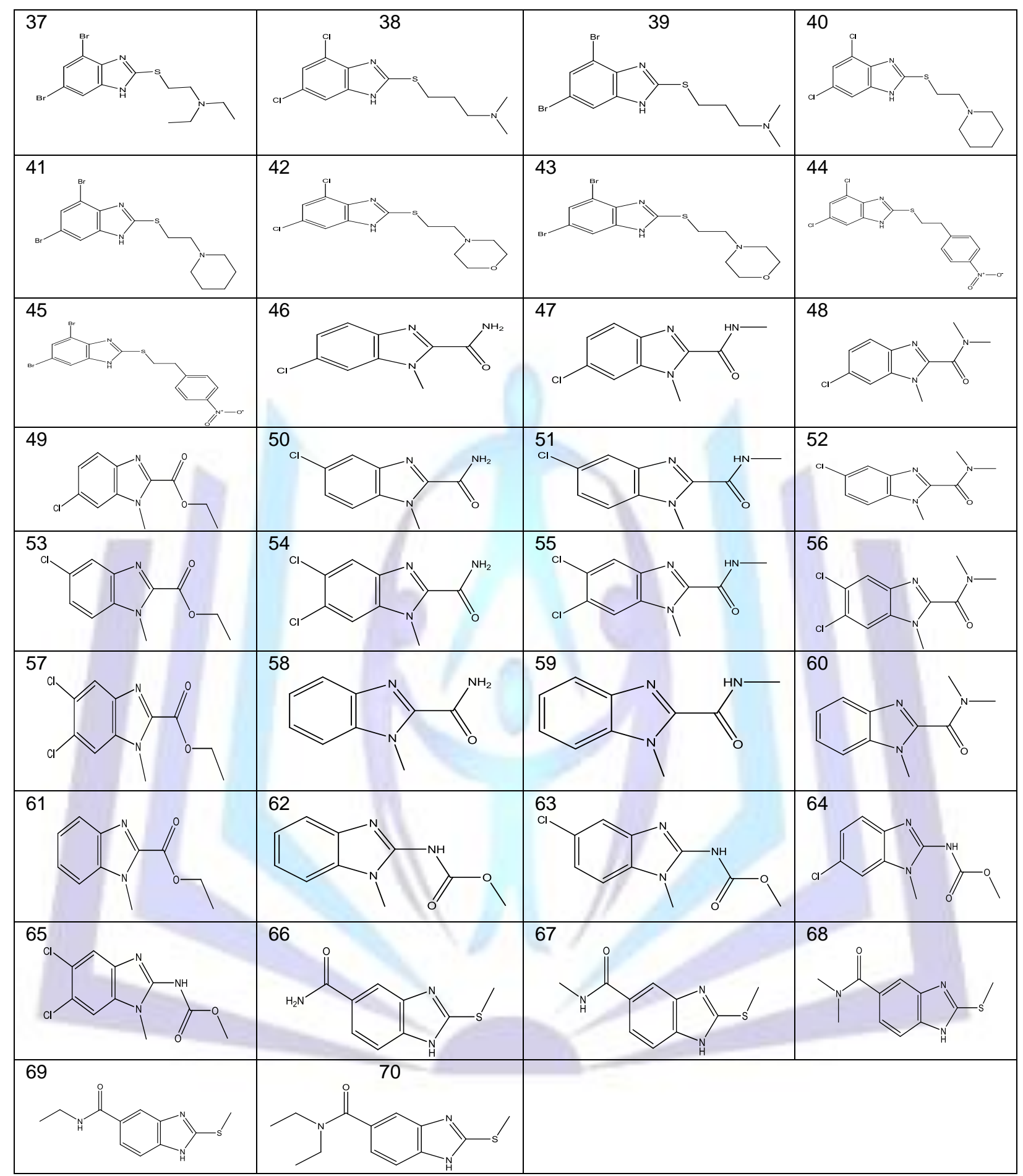


Table 2. The statistical parameters of different constructed QSAR models

\begin{tabular}{|l|c|c|c|}
\hline Pethod & Rarameters $_{\text {test }}$ & RMSE $_{\text {train }}$ & $\mathbf{R}^{{ }^{2}}$ \\
\hline GA-ANN Jack-Knife (gas) & 0.5051 & 0.1840 & 0.70 \\
\hline GA-ANN (gas) cross validation & 0.7316 & 0.1439 & - \\
\hline
\end{tabular}

Table 3. Experimental and predicted values of log (1//C50) using Jack-Knife method.

\begin{tabular}{|c|c|c|c|c|c|c|c|}
\hline \multicolumn{2}{|c|}{ Molecule } & \multicolumn{2}{|c|}{$\begin{array}{l}\text { Observed } \\
\log (1 / / C 50)\end{array}$} & \multicolumn{2}{|c|}{$\begin{array}{l}\text { Predicted } \\
\text { Jack-Knife }\end{array}$} & \multicolumn{2}{|c|}{$\begin{array}{c}\Delta \boldsymbol{\theta} \\
\text { Jack-Knife }\end{array}$} \\
\hline 1 & 36 & 5.500 & 7.390 & 5.6596 & 7.5133 & 0.1596 & 0.1233 \\
\hline 2 & 37 & 6.350 & 7.530 & 6.1778 & 7.8486 & 0.1722 & 0.3186 \\
\hline 3 & 38 & 5.500 & 7.070 & 5.7521 & 8.0758 & 0.2521 & 1.0058 \\
\hline 4 & 39 & 6.630 & 7.990 & 4.9851 & 6.7325 & 1.6449 & 1.2575 \\
\hline 5 & 40 & 5.640 & 7.140 & 5.0489 & 6.6407 & 0.5911 & 0.4993 \\
\hline 6 & 41 & 5.390 & 7.690 & 4.6203 & 7.8830 & 0.7697 & 0.1930 \\
\hline 7 & 42 & 5.270 & 7.290 & 5.4971 & 7.2359 & 0.2271 & 0.0541 \\
\hline 8 & 43 & 6.460 & 7.230 & 7.0341 & 7.2593 & 0.5741 & 0.0293 \\
\hline 9 & 44 & 6.700 & 7.960 & 5.2236 & 8.7301 & 1.4764 & 0.7701 \\
\hline 10 & 45 & 5.590 & 8.480 & 5.8807 & 8.0535 & 0.2907 & 0.4265 \\
\hline 11 & 46 & 4.550 & 6.960 & 5.0208 & 6.6984 & 0.4708 & 0.2616 \\
\hline 12 & 47 & 4.530 & 6.980 & 4.8003 & 6.9558 & 0.2703 & 0.0242 \\
\hline 13 & 48 & 4.970 & 6.630 & 4.9427 & 6.6072 & 0.0273 & 0.0228 \\
\hline 14 & 49 & 6.440 & 7.720 & 6.8496 & 7.0862 & 0.4096 & 0.6338 \\
\hline 15 & 50 & 6.520 & 6.730 & 6.9146 & 6.6625 & 0.3946 & 0.0675 \\
\hline 16 & 51 & 6.540 & 6.450 & 4.3453 & 6.6933 & 2.1947 & 0.2433 \\
\hline 17 & 52 & 6.710 & 6.680 & 5.7913 & 6.4232 & 0.9187 & 0.2568 \\
\hline 18 & 53 & 6.690 & 7.570 & 7.4746 & 7.0281 & 0.7845 & 0.5419 \\
\hline 19 & 54 & 6.790 & 6.870 & 6.9099 & 6.6415 & 0.1199 & 0.2285 \\
\hline 20 & 55 & 6.710 & 6.650 & 6.7043 & 6.6977 & 0.0057 & 0.0477 \\
\hline 21 & 56 & 6.870 & 7.120 & 6.9996 & 6.6515 & 0.1296 & 0.4685 \\
\hline 22 & 57 & 7.030 & 7.530 & 7.5474 & 7.2970 & 0.5174 & 0.2330 \\
\hline 23 & 58 & 6.980 & 6.780 & 7.0607 & 6.7669 & 0.0807 & 0.0131 \\
\hline 24 & 59 & 5.800 & 6.980 & 7.0071 & 6.8356 & 1.2071 & 0.1444 \\
\hline 25 & 60 & 6.660 & 6.370 & 5.4246 & 6.5479 & 1.2354 & 0.1779 \\
\hline 26 & 61 & 6.720 & 7.070 & 6.6052 & 7.1918 & 0.1148 & 0.1218 \\
\hline 27 & 62 & 6.570 & 5.880 & 7.4969 & 6.0867 & 0.9269 & 0.2067 \\
\hline 28 & 63 & 8.700 & 6.360 & 6.4421 & 4.6311 & 2.2579 & 1.7289 \\
\hline 29 & 64 & 6.520 & 6.460 & 5.9910 & 6.3281 & 0.5290 & 0.1319 \\
\hline 30 & 65 & 6.240 & 6.270 & 7.8581 & 6.3517 & 1.6181 & 0.0817 \\
\hline 31 & 66 & 5.000 & 6.670 & 8.2423 & 6.0290 & 3.2423 & 0.6410 \\
\hline 32 & 67 & 6.160 & 6.790 & 6.9014 & 7.1973 & 0.7414 & 0.4073 \\
\hline 33 & 68 & 6.830 & 7.540 & 6.8411 & 6.6210 & 0.0111 & 0.9190 \\
\hline 34 & 69 & 7.140 & 7.260 & 6.4149 & 7.3575 & 0.7251 & 0.0975 \\
\hline 35 & 70 & 6.510 & 6.050 & 5.7899 & 7.4083 & 0.7201 & 1.3583 \\
\hline
\end{tabular}


Table 4. Descriptors values by stepwise MLR model for correlation 0.4 to up.

\begin{tabular}{|c|c|c|c|c|c|c|}
\hline Molecule & BLI & RDF090m & $\mathbf{G}(\mathbf{N} . \mathbf{N})$ & MATS5v & RDF035u & HATS1e \\
\hline 1 & 0.770 & 0.000 & 0.000 & -0.777 & 8.632 & 0.309 \\
\hline 2 & 0.814 & 0.000 & 0.000 & -0.618 & 9.205 & 0.307 \\
\hline 3 & 0.738 & 0.000 & 0.000 & -0.644 & 7.425 & 0.316 \\
\hline 4 & 0.720 & 0.007 & 0.000 & -0.449 & 6.741 & 0.299 \\
\hline 5 & 0.745 & 2.750 & 12.084 & -0.556 & 7.876 & 0.306 \\
\hline 6 & 0.744 & 3.562 & 0.000 & -0.378 & 9.644 & 0.274 \\
\hline 7 & 0.744 & 0.000 & 0.000 & -0.316 & 9.661 & 0.267 \\
\hline 8 & 1.031 & 3.195 & 0.000 & -0.311 & 8.252 & 0.239 \\
\hline 9 & 1.039 & 2.008 & 0.000 & -0.288 & 11.449 & 0.228 \\
\hline 10 & 1.039 & 1.478 & 0.000 & -0.243 & 10.103 & 0.182 \\
\hline 11 & 0.798 & 2.394 & 0.000 & -0.172 & 14.556 & 0.236 \\
\hline 12 & 0.814 & 2.844 & 0.000 & -0.159 & 15.661 & 0.200 \\
\hline 13 & 0.814 & 1.160 & 0.000 & -0.140 & 16.225 & 0.209 \\
\hline 14 & 0.854 & 0.000 & 0.000 & 0.000 & 6.172 & 0.170 \\
\hline 15 & 0.893 & 0.000 & 0.000 & 0.250 & 6.745 & 0.242 \\
\hline 16 & 0.835 & 0.000 & 0.000 & -1.000 & 5.578 & 0.281 \\
\hline 17 & 0.940 & 0.000 & 0.000 & 0.440 & 6.607 & 0.175 \\
\hline 18 & 0.907 & 0.000 & 0.000 & -0.375 & 3.915 & 0.380 \\
\hline 19 & 0.938 & 0.000 & 0.000 & -0.083 & 6.149 & 0.241 \\
\hline 20 & 0.885 & 0.000 & 0.000 & -0.656 & 5.514 & 0.274 \\
\hline 21 & 0.981 & 0.000 & 0.000 & 0.047 & 5.985 & 0.173 \\
\hline 22 & 1.174 & 0.009 & 0.000 & 0.067 & 5.510 & 0.262 \\
\hline 23 & 0.929 & 0.000 & 0.000 & -0.556 & 5.455 & 0.271 \\
\hline 24 & 0.897 & 0.000 & 0.000 & -0.452 & 5.511 & 0.305 \\
\hline 25 & 1.009 & 0.000 & 0.000 & -0.348 & 5.529 & 0.298 \\
\hline 26 & 1.009 & 0.000 & 0.000 & -0.282 & 5.448 & 0.304 \\
\hline 27 & 1.110 & 0.000 & 0.000 & -0.257 & 5.385 & 0.297 \\
\hline 28 & 1.199 & 0.000 & 0.000 & -0.066 & 5.322 & 0.295 \\
\hline 29 & 0.818 & 1.188 & 0.000 & -0.477 & 6.351 & 0.257 \\
\hline 30 & 0.689 & 0.001 & 22.089 & -0.283 & 12.888 & 0.320 \\
\hline 31 & 1.117 & 10.969 & 0.000 & -0.091 & 9.635 & 0.324 \\
\hline 32 & 1.131 & 0.032 & 0.000 & 0.892 & 6.680 & 0.200 \\
\hline 33 & 1.286 & 0.035 & 0.000 & 0.396 & 6.737 & 0.195 \\
\hline 34 & 1.154 & 2.445 & 11.081 & 0.824 & 8.702 & 0.223 \\
\hline 35 & 1.292 & 8.763 & 11.079 & 0.231 & 8.799 & 0.219 \\
\hline
\end{tabular}


Table 4. Continued.

\begin{tabular}{|c|c|c|c|c|c|c|}
\hline Molecule & BLI & RDF090m & $\mathbf{G}(\mathbf{N} . . \mathbf{N})$ & MATS5v & RDF035u & HATS1e \\
\hline 36 & 1.212 & 4.858 & 11.114 & 0.845 & 11.788 & 0.213 \\
\hline 37 & 1.336 & 10.554 & 11.112 & 0.225 & 11.840 & 0.210 \\
\hline 38 & 1.172 & 3.712 & 12.559 & -0.032 & 11.161 & 0.188 \\
\hline 39 & 1.303 & 7.388 & 12.958 & -0.181 & 13.479 & 0.202 \\
\hline 40 & 1.181 & 2.789 & 11.059 & 0.834 & 13.516 & 0.191 \\
\hline 41 & 1.294 & 8.541 & 11.058 & 0.222 & 13.571 & 0.187 \\
\hline 42 & 1.124 & 3.391 & 11.090 & 0.433 & 8.631 & 0.212 \\
\hline 43 & 1.237 & 9.326 & 11.085 & 0.161 & 8.667 & 0.208 \\
\hline 44 & 1.017 & 3.645 & 19.861 & -0.041 & 11.425 & 0.147 \\
\hline 45 & 1.117 & 4.472 & 19.861 & -0.086 & 11.394 & 0.145 \\
\hline 46 & 0.878 & 0.000 & 6.546 & -0.331 & 7.230 & 0.228 \\
\hline 47 & 0.910 & 0.001 & 6.546 & -0.189 & 10.063 & 0.214 \\
\hline 48 & 0.921 & 0.023 & 6.546 & -0.109 & 14.653 & 0.187 \\
\hline 49 & 0.935 & 0.000 & 0.000 & -0.289 & 9.782 & 0.203 \\
\hline 50 & 0.878 & 0.248 & 6.546 & -0.409 & 7.814 & 0.230 \\
\hline 51 & 0.910 & 0.248 & 6.546 & -0.233 & 10.055 & 0.217 \\
\hline 52 & 0.921 & 0.047 & 6.546 & -0.137 & 12.731 & 0.188 \\
\hline 53 & 0.935 & 0.091 & 0.000 & -0.332 & 9.791 & 0.206 \\
\hline 54 & 0.914 & 0.248 & 6.546 & -0.325 & 7.751 & 0.228 \\
\hline 55 & 0.941 & 0.248 & 6.546 & -0.209 & 9.992 & 0.216 \\
\hline 56 & 0.950 & 0.265 & 6.681 & -0.138 & 10.763 & 0.226 \\
\hline 57 & 0.964 & 0.006 & 0.000 & -0.288 & 9.729 & 0.206 \\
\hline 58 & 0.838 & 0.000 & 6.546 & -0.455 & 7.878 & 0.229 \\
\hline 59 & 0.875 & 0.086 & 6.760 & -0.218 & 7.763 & 0.199 \\
\hline 60 & 0.889 & 0.094 & 6.678 & -0.103 & 12.055 & 0.223 \\
\hline 61 & 0.904 & 0.085 & 0.000 & -0.346 & 9.839 & 0.203 \\
\hline 62 & 0.841 & 0.134 & 0.000 & -0.706 & 9.794 & 0.226 \\
\hline 63 & 0.876 & 0.039 & 0.000 & -0.635 & 9.465 & 0.223 \\
\hline 64 & 0.876 & 0.261 & 0.000 & -0.569 & 9.531 & 0.221 \\
\hline 65 & 0.908 & 0.007 & 0.000 & -0.531 & 11.92 & 0.269 \\
\hline 66 & 1.053 & 0.191 & 10.968 & 0.390 & 9.777 & 0.241 \\
\hline 67 & 1.074 & 0.217 & 10.968 & 0.397 & 12.720 & 0.229 \\
\hline 68 & 1.076 & 0.077 & 10.968 & 0.398 & 15.368 & 0.191 \\
\hline 69 & 1.109 & 0.411 & 10.968 & 0.398 & 14.421 & 0.212 \\
\hline 70 & 1.144 & 0.106 & 10.968 & 0.392 & 14.087 & 0.159 \\
\hline
\end{tabular}

\section{CONCLUSION}

In our study, the linear methods were used to select the most significant descriptors. The GA-stepwise MLR and GA-ANN were used to construct a quantitative relation between the activities of benzimidazole derivatives and their calculated descriptors. GA-ANN has been successfully used for finding a QSAR model for benzimidazole derivatives. It provides the best results in comparison with other studied methods. The results of the Jack-Knife, compounds No. 20, 33, 58, 48 and 47 have the smallest difference between the observed and predicted values exist and are proposed for drug design.

\section{ACKNOWLEDGMENTS}

The authors thank the Research vice Presidency of Islamic Azad University, Rasht Branch, for their encouragement, permission, and financial support.

\section{REFERENCES}

[1] Workowski KA., Berman S. MMWR Recomm Rep., 59: 1-110 (2010).

[2] Sutton M., Sternberg M., Koumans EH., McQuillan G., Berman S., Markowitz L., Clin Infect Dis., 45: 1319-1326 (2007).

[3] Barker HA., Smyth RD., Weissbach H., Toohey JI., Ladd JN., Volcani BE., Journal of Biological Chemistry., 235: 480 488 (1960).

[4] Demchuk E., Ruiz P., Chou S., Fowler B.A., SAR/QSAR methods in public health practice., Toxicol. Appl. Pharmacol., 254: 192-197 (2011). 
[5] Tsakovska I., Pajeva I., Alov P., Worth A., Recent advances in the molecular modeling of estrogen receptor-mediated toxicity., Adv. Protein Chem. Struct. Biol., 85: 217-251 (2011).

[6] Prasanna S., Manivannan E., Chaturvedi S.C., Bioorg. Med. Chem. Lett., 14: 4005 (2004).

[7] Prasanna S., Manivannan E., Chaturvedi S.C., QSAR Comb. Sci., 23: 621 (2004).

[8] Prasanna S., Manivannan E., Chaturvedi S.C., Arch. Pharm. Pharm. Med. Chem., 337: 440 (2004).

[9] Prasanna S., Manivannan E., Chaturvedi S.C.. Bioorg. Med. Chem. Lett., 15: 313 (2005).

[10] Prasanna S., Manivannan E., Chaturvedi S.C., Bioorg. Med. Chem. Lett., 15: 2097 (2005).

[11] Prasanna S., Manivannan E., Chaturvedi S.C., Indian J. Chem. B., 43: 2249 (2004).

[12] Manivannan E., Prasanna S., Chaturvedi S.C., Indian J. Biochem. Biophys., 41: 179 (2014).

[13] He L., Jurs P.C., J. Molecular Graphics and Modelling., 23: 503-523 (2005).

[14] Eldred D. V., Weikel C. L., Jurs P.C., Kaiser K.L.E., Chem. Res. Toxicol., 12: 670-678 (1999).

[15] Pérez-Villanueva J.L., Medina-Franco J.R., Caulfield Th., Hernández-Campos A., Hernández-Luis F., Yépez-Mulia L., Castillo R., European Journal of Medicinal Chemistry., 46: 3499-3508 (2011).

\section{Author' biography with Photo}

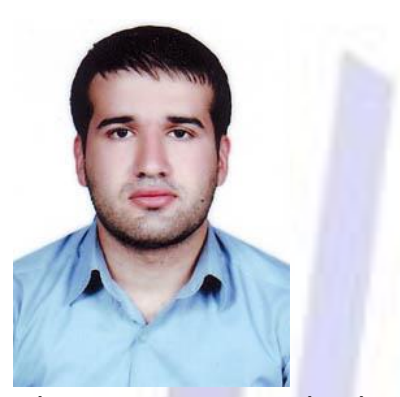

Alireza Nemati Rashtehroodi received his BSc degree in Chemistry in 2009, MSc in Organic Chemistry in 2012 from Payame Noor University, Sari, Iran and Master's thesis did in Chemometrics field from Payame Noor University, Sari, Iran in 2012 under supervision of Dr. Ghasem Ghasemi and Dr. Sattar Arshadi. His research fields include: chemometrics (Quantitative Structure - Activity Relationships) and nanothechnology in Computational chemistry, study of drug delivery.

\section{Education}

Payame Noor University, Sari Branch

Master of Science (M.Sc), organic Chemistry, A

$2010-2012$

Activities and Societies: Member of the University Research Committee(2005-2009)/ Member of journal of "Synthesis" as an editor and writer (2005-2009)/ Chairman of the Executive Committee Student Student Regional Conference of Chemistry (2008)/ President of the Scientific Society of Chemistry (2008)/ Member of Iranian Chemical Society (2013-now)/ Member ofYoung Researchers Club and Elite (2011-now)/ Member of Quality Control Officials Association of Guilan (2013- now)/ Member of Society Food, Cosmetics province of Guilan (2013-now).

Yours sincerely,

Alireza Nemati Rashtehroodi 